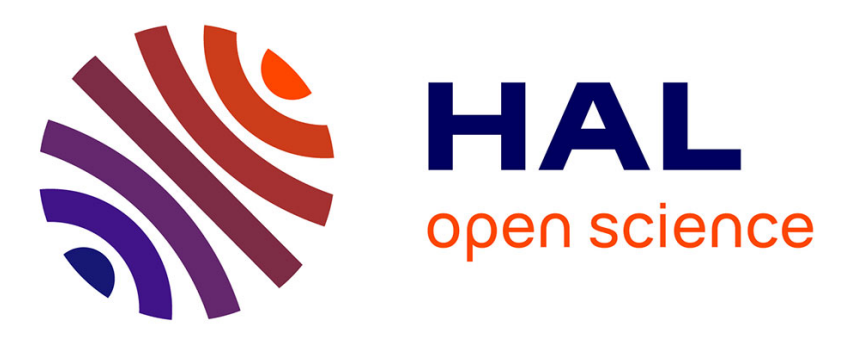

\title{
Le nucléaire fait son cinéma
}

Aurélien Portelli, Franck Guarnieri, Christophe Martin

\section{To cite this version:}

Aurélien Portelli, Franck Guarnieri, Christophe Martin. Le nucléaire fait son cinéma. Revue Generale Nucleaire, 2014, janvier-février (1), pp.96-101. hal-00971690

\section{HAL Id: hal-00971690 \\ https: / hal-mines-paristech.archives-ouvertes.fr/hal-00971690}

Submitted on 17 Dec 2014

HAL is a multi-disciplinary open access archive for the deposit and dissemination of scientific research documents, whether they are published or not. The documents may come from teaching and research institutions in France or abroad, or from public or private research centers.
L'archive ouverte pluridisciplinaire HAL, est destinée au dépôt et à la diffusion de documents scientifiques de niveau recherche, publiés ou non, émanant des établissements d'enseignement et de recherche français ou étrangers, des laboratoires publics ou privés. 


\title{
Le nucléaire fait son cinéma
}

\author{
aurélien PORTELlI, Franck GUARNIERI et Christophe MARTIN, Centre de Recherche sur les \\ Risques et les Crises (CRC) - MINES ParisTech
}

Pour le chercheur, le film, et plus particulièrement les fictions du cinéma, est une source qui participe à la compréhension des phénomènes passés, présents et à venir. II inscrit des usages, des valeurs, mais aussi des non-dits et des lapsus, qui témoignent d'une vision à la fois fidèle et déformée de la réalité sociale. Le miroir filmique projette une image reconstruite du Monde ; mais l'image n'est pas une simple projection, car elle contribue aussi à l'élaboration des processus sociaux. Le film diffuse des représentations qui peuvent influencer l'opinion et le comportement du public. II invite également le chercheur à s'interroger sur sa discipline et ses pratiques.

\section{Introduction}

Tout est affaire de représentations. Le nucléaire n'échappe pas à ce constat. La notion de représentation recouvre ainsi l'ensemble des opinions, des croyances, des mythes qui sont produits et partagés par les membres d'un groupe, à partir d'un objet ou d'un système particulier. Ceux-ci peuvent concerner des enjeux forts et se trouver au centre d'une controverse, comme c'est le cas pour le nucléaire (Martin et al., 2013). L'analyse des représentations du nucléaire se justifie donc pleinement du fait de l'impact déterminant qu'elles ont sur le débat public, et la prise de décision politique. Les sources de la connaissance concernent dès lors des domaines aussi divers que la peinture, la photographie, la littérature, les articles et les dessins de presse, la publicité, les documents audiovisuels, et parmi ces derniers les films.

L'objet filmique occupe une place particulière dans I'histoire des représentations, lié à son potentiel de diffusion médiatique et de "colonisation" des consciences. II s'est emparé de l'atome de plusieurs manières : par le film publicitaire (Notre ami l'atome, Disney, 1957), les actualités ("Marcoule", Actualités françaises, 1955), le documentaire (Centrales nucléaires : démantèlement impossible ?, B. Nicolas, 2013), la série télévisée (Les Simpson, M. Groening), le téléfilm (Ouragan nucléaire, F. Olen Ray, 2007), le film technique (Protection de l'intervention sur le

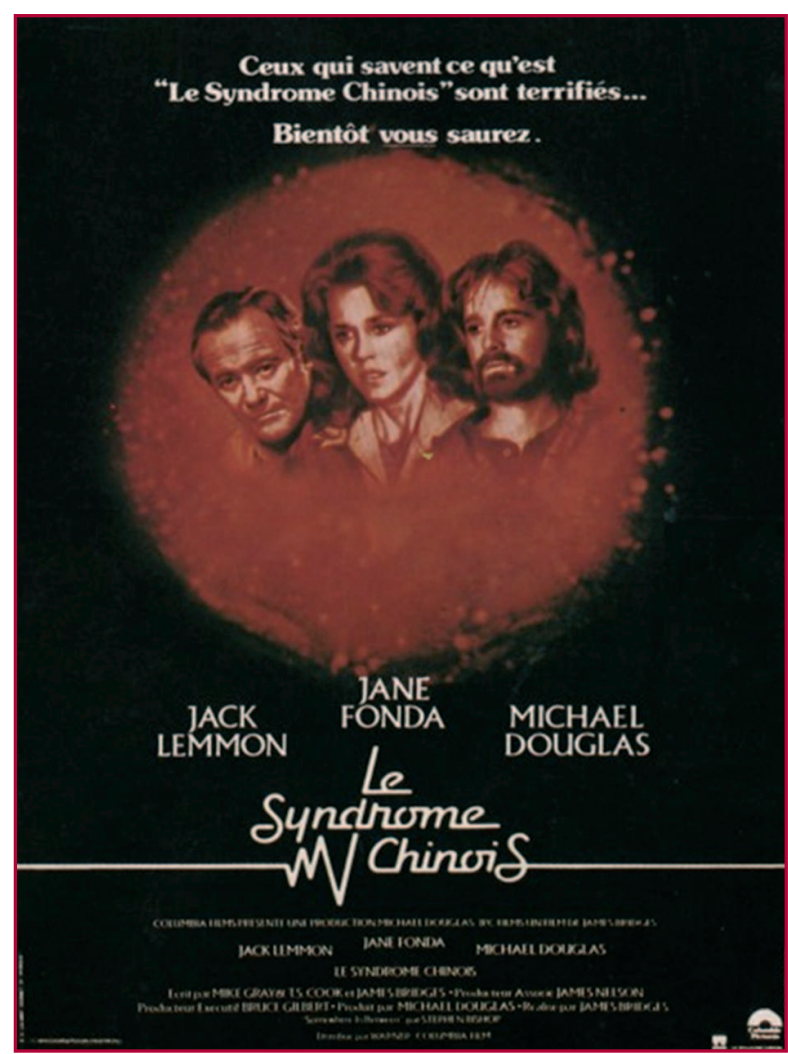

Le syndrome chinois (J. Bridges, 1979) : Il s'agit du film le plus célèbre sur la filière. Une journaliste et son cameraman réalisent un reportage dans la centrale de Ventana. Une secousse sismique cause un incident, que les personnages parviennent à filmer clandestinement. La fusion du cœur du réacteur est évitée, mais l'exploitant refuse de vérifier l'état de l'installation avant sa remise en service. Douze jours après la sortie du film aux Etats-Unis, un accident se produit dans la centrale de Three Mile Island (28 mars 1979). Cette coïncidence, qui marque durablement le public, a contribué au succès du Syndrome chinois.

canal W-19-E, SPR de Marcoule) et la fiction cinématographique. Du point de vue du cinéma, la représentation implique deux moments qui sont liés: "le passage d'un texte, écrit ou non, à sa matérialisation par des actions dans des lieux agencés en scénographie (temps de la mise en scène) ; le passage de cette représentation analogue à celle du théâtre, à une image en mouvement, par le choix de cadrages et la construction d'une séquence d'images (montage)" (J. Aumont, M. Marie, 2008, p. 176). 
L'objectif de cet article est donc de montrer l'intérêt d'étudier les représentations cinématographiques sur le nucléaire qui révèlent et façonnent le débat public. La première partie de cette étude présente succinctement la filmographie consacrée à l'usage militaire et civil de l'atome. La deuxième partie met en perspective l'approche thématique et monographique des représentations cinématographiques. Cette dernière est illustrée, dans la troisième partie, par une brève analyse de Grand Central, film réalisé en 2013 par Rebecca Zlotowski, qui décrit les conditions de travail des sous-traitants du nucléaire.

\section{La filmographie sur le nucléaire}

Le thème de la radioactivité est abordé par les cinéastes quelques années avant la Seconde Guerre mondiale. L'utilisation de la bombe A par les Etats-Unis suscite une vague de films, accentuée par les tensions militaires de la Guerre froide. Les scénarios transposent les angoisses des populations, telles que le risque d'un conflit nucléaire entre les grandes puissances, l'anéantissement de l'humanité ou les mutations dues aux radiations. Ces représentations apparaissent dans de nombreux genres : le fantastique (Godzilla, I. Honda, 1954), le drame (Hiroshima mon amour, A. Resnais, 1959), la comédie (Docteur Folamour, S. Kubrick, 1964), le film d'espionnage (Goldfinger, G. Hamilton, 1964), la science-fiction

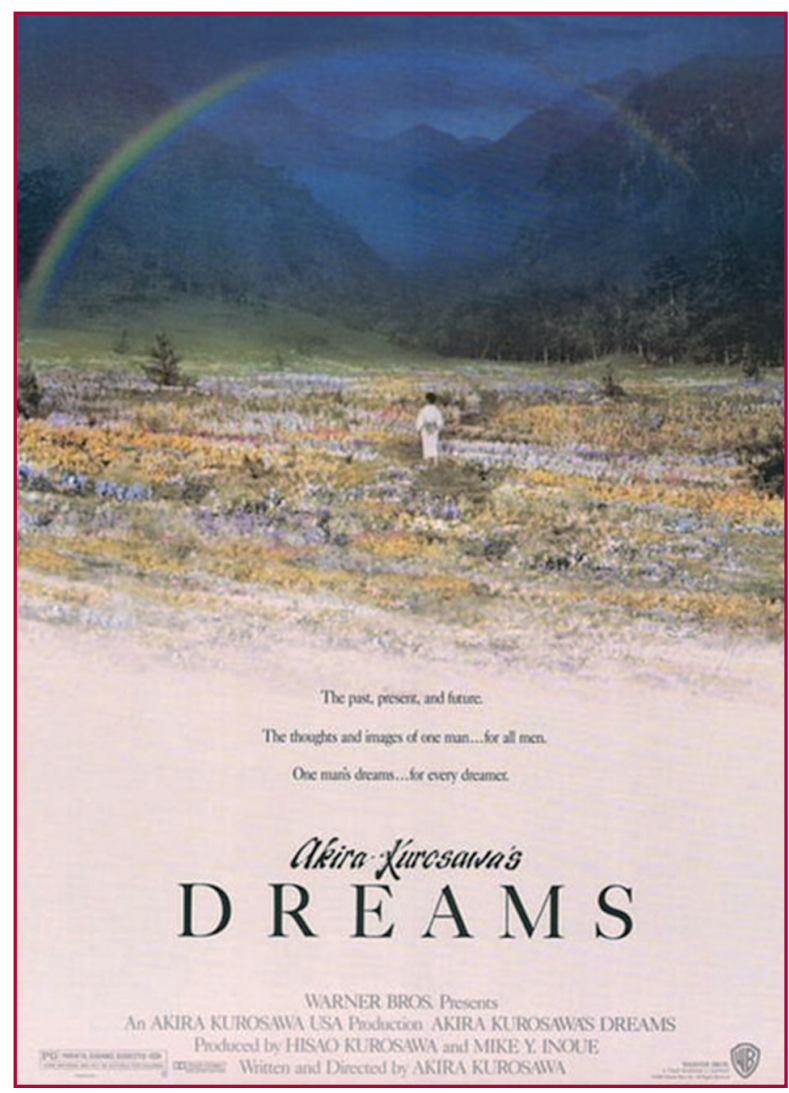

Le Mont Fuji en Rouge (court-métrage présenté dans Rêves, A. Kurosawa, 1990) : L'éruption du Mont Fuji provoque des accidents en chaîne dans les centrales nucléaires japonaises. La population, affolée, tente de s'enfuir. Les survivants, bloqués face à la mer, se retrouvent confrontés aux radiations qui contaminent l'archipel.
(Terminator, J. Cameron, 1984) ou le film historique (Les maitres de l'ombre, R. Joffé, 1989). La représentation apocalyptique de l'atome, étudiée depuis longtemps par les chercheurs (H. Puiseux, 1987), a ainsi alimenté l'imaginaire des spectateurs et influencé le regard porté sur cette technologie.

La filmographie consacrée à l'atome civil est bien plus courte. Quelques œuvres remarquables permettent néanmoins d'ouvrir un chantier de recherche, sans pour autant viser l'exhaustivité. Le syndrome chinois (J. Bridges, 1979), Le Mont Fuji en Rouge (A. Kurosawa, 1990), La terre outragée (M. Boganim, 2012), The Land of Hope (S. Sion, 2012) et Grand Central (R. Zlotowski, 2013) constituent ainsi le corpus de films sur lequel se fonde notre analyse des représentations nucléaires.

Cet ensemble de films peut être divisé en deux catégories. La première regroupe les fictions dont certaines séquences se déroulent dans une centrale nucléaire. Les films concernés sont Le syndrome chinois et Grand Central. Leur intérêt est de reconstituer des situations de travail et de présenter une description parfois très négative de l'organisation. Ainsi, l'exploitant est considéré, dans Le syndrome chinois, comme une entreprise sans scrupules, qui se préoccupe davantage de ses bénéfices que de la sûreté des installations et de la protection des populations.

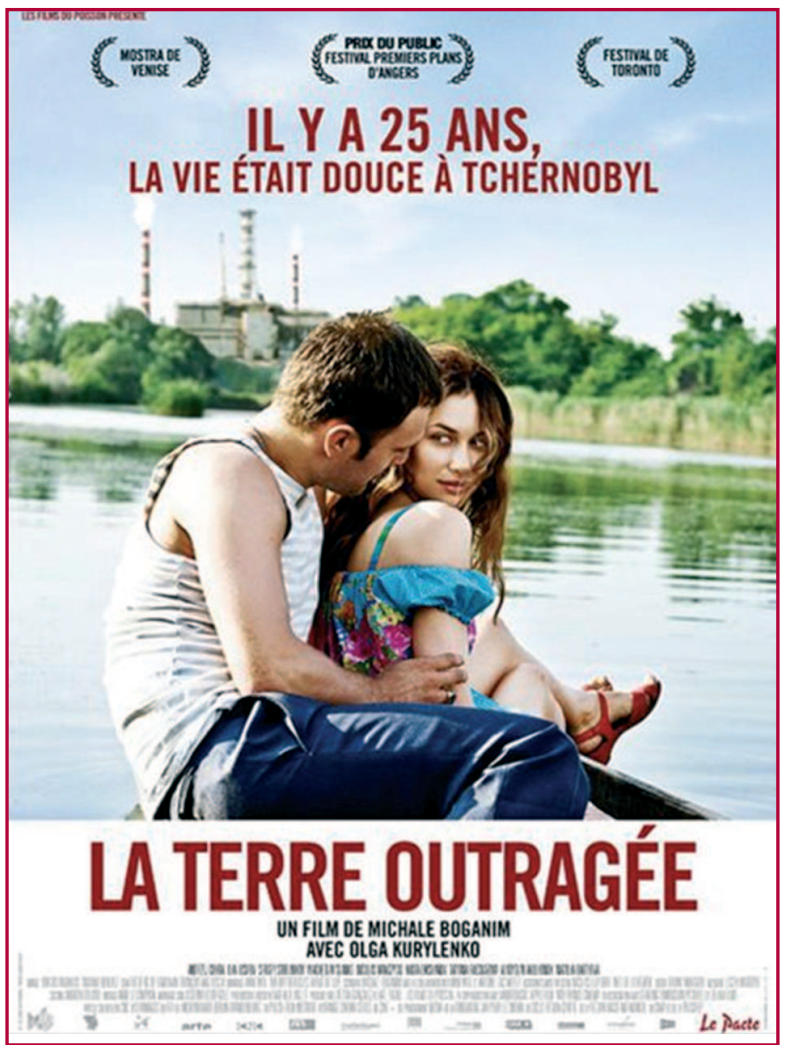

La terre outragée (M. Boganim, 2012) : Anya épouse un pompier la veille de l'accident de Tchernobyl. II est mortellement irradié lorsqu'il intervient, le lendemain, pour éteindre l'incendie autour de la centrale. Dix ans plus tard, la jeune femme est devenue guide dans la zone interdite, où des visites sont désormais organisées. 
La seconde catégorie rassemble les films qui traitent le sujet du nucléaire sans pour autant montrer ce qui se fait dans la centrale. Le Mont Fuji, La terre outragée et The Land of Hope évacuent par conséquent toute dimension opérationnelle et organisationnelle, pour décrire le sort des victimes de la catastrophe nucléaire. La centrale est reléguée à un élément de l'arrière-plan dans La terre outragée, tandis qu'elle n'est pas montrée dans The Land of Hope. La caméra ne s'aventure pas dans la centrale, confinée dans le hors-champ.

Chaque catégorie observe donc la filière sous un angle particulier : le travail et l'exploitant d'un côté, l'accident majeur et les sinistrés de l'autre. Cette typologie, sans pour autant faire apparaître de "nouveaux" genres cinématographiques, permet au chercheur d'organiser son analyse des représentations, qui peut être menée de différentes manières.

\section{L'analyse des représentations cinématographiques}

Le chercheur considère le film comme une source qui participe à la compréhension de la réalité sociale. Le cinéma exerce également une influence sur "les publics" - au sens où la réception d'un film évolue selon le lieu et l'époque où il est visionné : "si les films peuvent infléchir les comportements individuels ou collectifs, ce n'est pas parce qu'ils offrent à la société un miroir dans lequel son image serait renvoyée. Ce qui les distingue, au contraire, c'est leur volonté inaugurale de se livrer à une reconstruction du présent comme du passé, et non à une reconstitution ou une simple duplication" (C. Delage et V. Guigueno, 2004, p. 13-14). Pour l'historien, la fiction inscrit dès lors des usages, des valeurs, mais aussi des non-dits et des lapsus (M. Ferro, 1993), qui témoignent d'une vision reconstruite du réel. La méthodologie qu'il utilise croise les acquis de l'analyse filmique et historique. La "lecture" interne du film n'est pas suffisante. II faut la compléter en intégrant la fiction à son contexte de production.

L'analyse des représentations peut être menée sous un angle thématique ou monographique. L'approche thématique permet de repérer, à partir des catégories précédemment décrites, des rubriques significatives, comme l'espace de la centrale, l'image de l'exploitant et du travailleur, les opérations de décontamination, l'impact de l'accident sur les populations, la radioactivité, etc. Dans Le Mont Fuji, de longues traînées rouges et violettes, chargées de particules radioactives, balaient un paysage de désolation. "La stupidité de l'homme est sans bornes. Ce n'est pas visible la radioactivité, alors on a développé une technologie qui la colore quand elle se répand dans l'air', annonce l'un des personnages. Akira Kurosawa dresse un constat alarmant : les progrès de la science ne servent plus à préserver la vie, mais à mieux percevoir la mort. Le court-métrage, sorti en 1990, préfigure, à travers une vision cauchemardesque, le risque d'un accident nucléaire au Japon. L'éruption du Mont Fuji, la montagne sacrée de l'archipel, provoque une réaction en chaîne qui punit l'hybris des hommes. Les représentations à l'œuvre - les centrales sont comparées à des bombes à retardement et les ingénieurs à des "apprentissorciers" au service d'une technique aussi dangereuse que contreproductive - nourrissent l'imaginaire de la fin du monde, qui traverse toutes les civilisations.
Deux décennies plus tard, Sono Sion réalise The Land of Hope pour évoquer les conséquences de Fukushima. Le réalisateur utilise, dans une séquence, le même procédé chromatique que Kurosawa. Izumi développe une phobie de la contamination radioactive lorsqu'elle apprend qu'elle est enceinte. En sortant de la clinique, elle a une vision qui la tétanise. Elle aperçoit, derrière la double porte vitrée, de la fumée rouge obscurcir entièrement la rue. Le rouge, symbole de l'enfer radioactif comme dans Le Mont Fuji, se reflète dans les yeux d'Izumi. Un clignement de paupière chasse l'illusion, mais la peur s'est désormais enracinée en elle.

L'analyse thématique présente l'avantage de comparer les films et de croiser les images entre elles, mais elle confronte le chercheur à une difficulté majeure : la filmographie sur la filière n'est pas suffisamment abondante pour se consacrer à un corpus national. L'approche thématique implique donc, dans le cadre de cette recherche, de comparer des films de nationalité différente (américaine, japonaise et française). Or, elle permet difficilement de contextualiser les représentations, puisque celles-ci sont dissociées de leur environnement culturel d'origine. A titre d'exemple, Le syndrome chinois, sorti aux Etats-Unis en 1979 avant Three Mile Island, et Grand Central, sorti en France en 2013 après Fukushima, répondent à des critères d'élaboration spécifiques, qu'il ne faut pas négliger. Etudier les films dans ces cas, nécessite de les replacer dans "leur" histoire, en étudiant les différentes

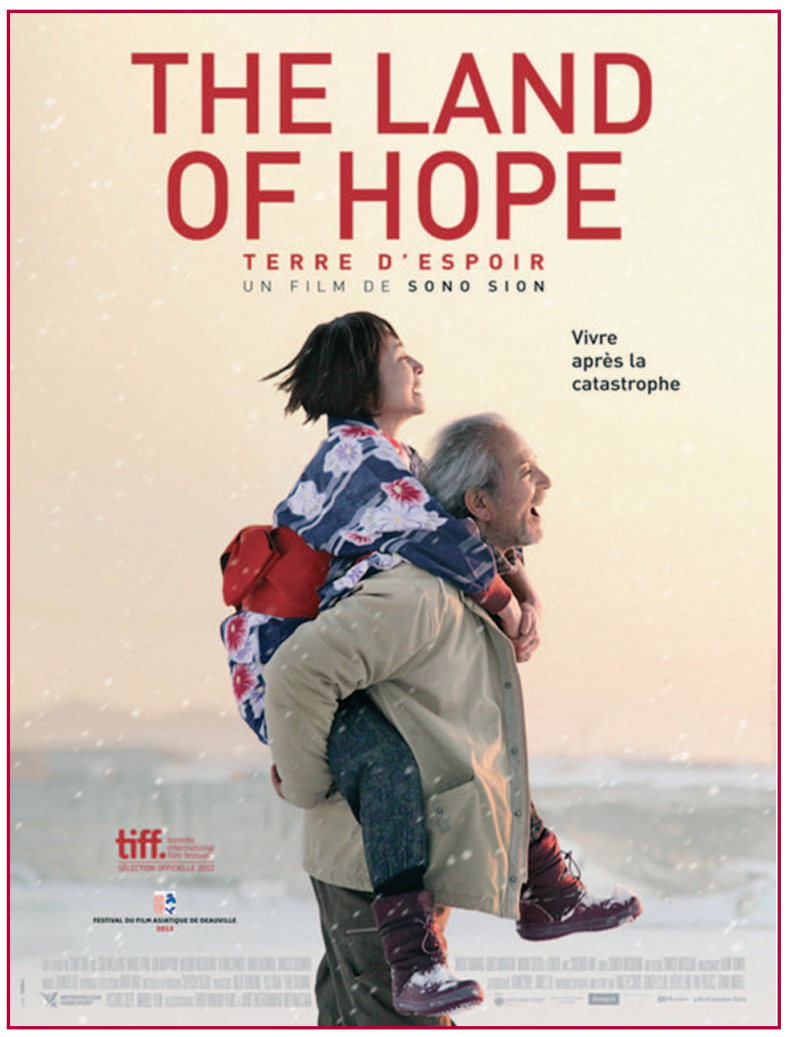

The Land of Hope (S. Sion, 2012) : Un accident majeur, causé par un tremblement de terre, a lieu à la centrale de Nagashima (contraction de Nagasaki-HiroshimaFukushima). Le film montre les conséquences de la catastrophe sur trois couples qui habitent dans une petite ville située près de la centrale. 
contraintes (conjoncturelles, idéologiques, narratives, etc.) de production, et la manière dont l'accident nucléaire a pu modifier le regard porté sur la filière.

L'approche monographique prend alors tout son sens. Elle consiste à analyser les films séparément, en les présentant par catégorie ou de manière chronologique. Elle permet de bien contextualiser chaque œuvre, en révélant ses conditions de production et de réception, ainsi que ses relations avec le débat public du moment. Une telle approche n'interdit nullement de s'intéresser au "dialogue" entre la représentation de l'atome civil et militaire. Par exemple, une séquence de La terre outragée présente un plan d'ensemble de la ville de Pripyat. Les toits des immeubles, recouverts par la neige, sont filmés en plongée. Un panoramique horizontal fait alors découvrir au public la vision d'un Pompéi soviétique. Cette image d'un monde fini et inhabité rejoint l'imaginaire post-apocalyptique véhiculé par les films abordant les ravages d'un conflit nucléaire.

L'analyse monographique n'interdit pas non plus d'établir ponctuellement des corrélations avec d'autres médias, comme le documentaire. Cette opportunité permet d'enrichir le travail de recherche, en établissant un réseau de représentations plus élaboré et complet. Une difficulté demeure cependant : la fiction et le documentaire ne sont pas de même nature. Ils présentent des caractéristiques bien distinctes et leur comparaison n'est pas toujours évidente. II ne s'agit donc pas de confronter la fiction au documentaire, mais de considérer ce dernier comme un élément de référence, pour mieux comprendre le cadre culturel auquel le film appartient.

Par conséquent, le chercheur a intérêt à privilégier la monographie pour étudier la représentation cinématographique de la filière. L'analyse de Grand Central a été choisie pour illustrer cette méthodologie pour plusieurs raisons. II s'agit tout d'abord de la première fiction française à s'intéresser d'aussi près aux hommes du nucléaire. Le film de Rebecca Zlotowski contient également des séquences de travail, qui permettent au spectateur de mieux comprendre la dimension opérationnelle de cette industrie, souvent mal connue. Le discours diffusé dans Grand Central présente enfin un parti pris évident sur l'exploitant et la sous-traitance, qui alimente l'imaginaire de l'atome sur fond de catastrophe et d'exploitation d'une main-d'œuvre précaire. Le film occupe donc une place importante dans l'histoire des représentations du nucléaire et participe de fait à façonner l'opinion publique.

\section{La représentation des travailleurs du nucléaire dans Grand Central}

Le film de Rebecca Zlotowski s'inscrit dans un imaginaire où la centrale est représentée comme un espace sacralisé. La réalisatrice fait dialoguer, selon ses propos, "la naissance d'un état amoureux et les manifestations de la radioactivité à l'intérieur d'un lieu aussi étrange qu'une centrale nucléaire" (Le Monde, 26 août 2013). La manière dont celle-ci est montrée à l'écran présente en effet un lieu qui n'a rien de banal. Les tours de refroidissement, éléments indissociables de l'esthétisation de l'œuvre, renvoient, tels les clochers d'une cathédrale, à la puissance mystérieuse contenue dans l'édifice. Cette impression est particulièrement ressentie lorsque Gary, le personnage principal, arrive pour la première fois à la centrale. La porte s'ouvre en faisant un grincement métallique et le protagoniste entre dans le bâtiment, comme on pénètre dans un sanctuaire. Le vestiaire ressemble à une sacristie, où les officiants se préparent avant d'accéder au cœur (chœur) du réacteur.

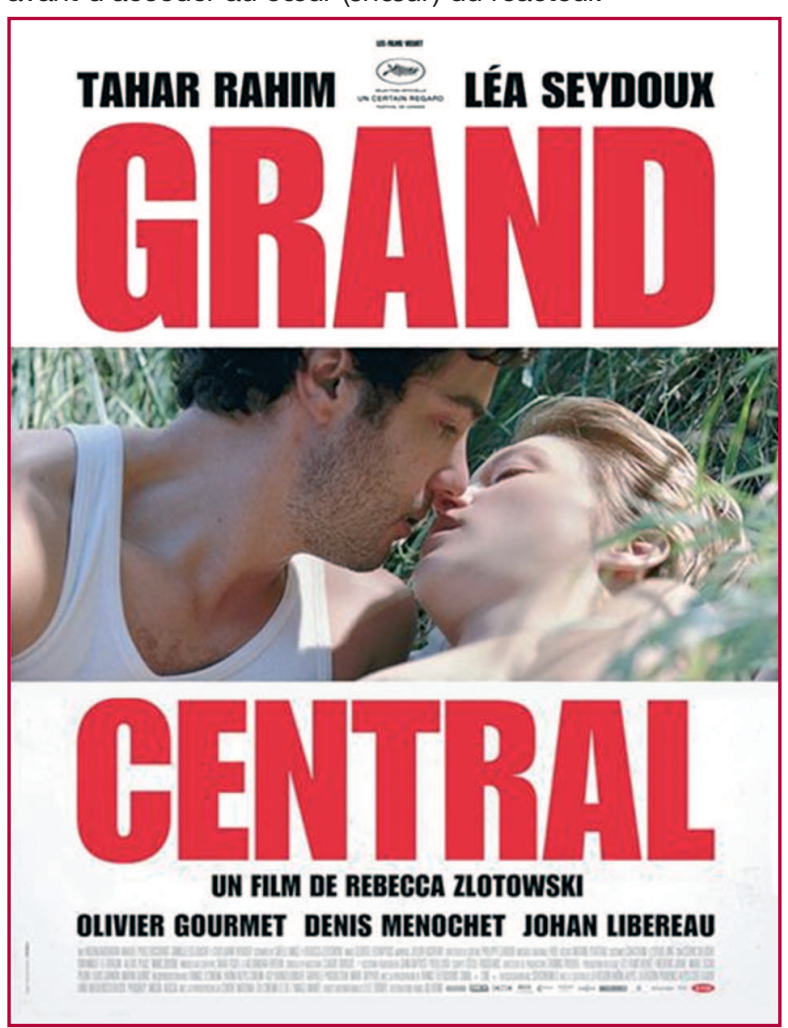

Grand Central (R. Zlotowski, 2013) : Gary n'a pas terminé son CAP en chaudronnerie et a enchaîné les emplois temporaires, avant d'être embauché dans une centrale nucléaire. Le travail est dangereux et éprouvant. Le protagoniste s'intègre à l'équipe de sous-traitants et a une relation secrète avec Karole, la compagne de son collègue Toni. Gary est lentement contaminé, à la fois par l'amour et les radiations.

Le film met en évidence la lutte contre la radioactivité, qui est abordée comme un personnage hors-champ. Les opérations de décontamination sont filmées ou évoquées dans les dialogues comme des joutes face à un ennemi invisible. Les métaphores animalières expriment toute la dangerosité de cette lutte. Gary chevauche un taureau mécanique, comme les travailleurs tentent de dompter l'atome. Le crocodile, aperçu lors d'une visite au zoo et qui fascine tant les protagonistes, est aussi sournois et imperceptible que la radiation; il peut rester immobile pendant des heures pour mieux surprendre sa proie. Le registre sacré, qui conduit à la fois l'architecture et les processus de travail (C. Bergé, 2010), rejoint ici une tradition de l'imaginaire atomique qui s'est imposée dès le début de cette industrie'1.

${ }^{1}$ Dans les années 1950, la presse et les autorités du Gard comparent le site du CEA Marcoule à une "cathédrale", capable de rivaliser avec les monuments les plus prestigieux de Paris (G. Hecht, 2004). 
Le film questionne les choix faits par l'exploitant, en dénonçant le manque de formation et les mauvaises conditions de travail des sous-traitants ${ }^{2}$. Pour comprendre cette représentation, il faut pouvoir la confronter à la réalité. L'objectif est non pas de déprécier la valeur de l'image - le cinéma n'a pas à reproduire le réel, il doit le "réinventer" - mais de mettre en exergue la spécificité de son message. Le scénario, qui s'inspire du roman La Centrale (E. Filhol, 2010), ré-explore le quotidien des sous-traitants dans un contexte post-Fukushima et repose la question du coût humain de la production d'énergie. Les rapports de pouvoir entre les entreprises exploitantes du nucléaire et les entreprises sous-traitantes sont réels et bien connus des chercheurs ${ }^{3}$. Pourtant, les sous-traitants tels qu'ils sont évoqués ne sont pas représentatifs de l'ensemble de cette communauté de travailleurs. Un recrutement expéditif, une formation de quelques jours en radioprotection et une simple visite médicale introduisent le personnage principal dans le monde de la sous-traitance. Ces étapes sont traitées comme des formalités de façade, qui discréditent d'emblée les procédures d'embauche. L'exploitant profiterait sciemment du manque de qualification du travailleur et mettrait délibérément sa santé en danger.

La dimension sociopolitique du film est très prononcée : un parking est réservé à l'aristocratie (le personnel d'EDF), un autre aux "trimardeurs du nucléaire" (les sous-traitants). Les personnages vivent à la périphérie de la centrale, dans des mobile-homes exigus et mal équipés. La difficulté des conditions de travail et de vie augmente la fréquence des accidents. La domination de l'exploitant prend forme lors des opérations de décontamination filmées par la réalisatrice. Les violences faites aux corps par les hommes des services de radioprotection évoquent en creux les univers concentrationnaires et la dépersonnalisation des travailleurs.

Ce discours sur la sous-traitance est fréquemment relayé par les médias, en témoignent l'article "Dans les centrales françaises, le malaise de la viande à radiations" (Libération, 21 mars 2011) ou le documentaire Nucléaire : la bombe humaine (E. Fayner, 2012) ${ }^{4}$. Si la main-d'œuvre précaire existe dans le nucléaire, la sous-traitance ne se résume pourtant pas à des salariés nomades surexposés aux radiations au nom de la rentabilité économique. Les travailleurs du secteur vivent une précarité liée aux contrats qui lient leur entreprise aux donneurs d'ordre, mais sont pour une large part d'entre eux salariés dans des contrats stables et sédentarisés.

Grand Central ne contextualise pas l'organisation des hommes du nucléaire dans la lutte syndicale, alors qu'ils sont nombreux à revendiquer leur savoir-faire et leur identité professionnelle. Les frontières ne sont pas aussi marquées entre les salariés de l'exploitant et ceux des sous-traitants. En témoigne la section syndicale mixte entre salariés d'EDF et salariés sous-traitants de la centrale de Cruas, précisément celle où l'action du film est censée se dérouler. Les équipes travaillent ensemble et la dépendance des agents du donneur d'ordre aux savoir-faire des sous-traitants est forte ${ }^{5}$.

La focalisation sur la précarité des hommes du nucléaire verrouille un discours qui ne permet pas de montrer qu'ils sont des acteurs à part entière de l'organisation. En effet, leurs interactions avec le donneur d'ordre reposent non pas sur de simples relations de dépendance, mais sur des stratégies qui conduisent aussi à des alliances (Martin et Guarnieri, 2012). Les choix opérés par le scénario sont donc révélateurs des enjeux actuels du débat public. Ils traduisent, à partir de la description des sous-traitants, les inquiétudes concernant les conditions de travail dans le secteur et plus implicitement la sûreté des centrales françaises.

\section{Conclusion}

Si l'atome civil a moins séduit les cinéastes que son application militaire, un ensemble de films se distingue néanmoins, et participe à la construction de l'opinion publique sur la question de l'énergie nucléaire. Le film de Rebecca Zlotowski, en évoquant la précarité des soustraitants, vient alimenter les débats sur l'énergie nucléaire et la sûreté des installations, ravivés depuis l'accident de Fukushima. Dans un contexte de prise de décision politique sur la sortie du nucléaire, ce film pèse d'un poids non négligeable sur le débat public. II s'inscrit dans la bataille des images dont l'issue pourrait bien déterminer la pérennité de la filière. Etudier les représentations véhiculées par les documentaires et les œuvres de fiction permet d'actualiser les connaissances que l'on a de l'imaginaire collectif et d'anticiper les éléments du débat. Dans la confrontation des idées sur la transition énergétique, le cinéma donne une longueur d'avance aux partisans de l'arrêt des centrales. Le genre cinématographique opère une transfiguration du réel infiniment plus séduisante qu'un discours techniciste sur la réalité de la filière. Discuter ces représentations s'avère alors nécessaire pour assurer les conditions d'un débat démocratique.

\section{Références bibliographiques}

Aumont J., Marie M. (2008). Dictionnaire théorique et critique du cinéma. Paris, Armand Colin, 300 p.

\footnotetext{
${ }^{2}$ Les sous-traitants interviennent lors des arrêts de tranche des centrales pour effectuer des opérations de maintenance qui ne sont pas réalisées par les agents de l'exploitant.

${ }^{3}$ Annie Thébaud-Mony a ainsi évoqué la gestion des ressources par la dose et sous-titré son ouvrage consacré à l'industrie nucléaire, "Sous-traitance et servitude" (2000). Cette vision dominée du salarié sous-traitant n'est d'ailleurs pas propre au nucléaire et Michael Quinlan, chercheur australien, évoque l'externalisation des risques lors des opérations de sous-traitance (1997).

${ }^{4}$ Les entretiens évoquent la course à la rentabilité et les risques sanitaires qui pèsent sur les travailleurs : "La santé des sous-traitants dans le monde de l'atome demeure un tabou. Aucun chiffre officiel, pas d'études scientifiques. Les hommes sont invisibles".

${ }^{5}$ Les salariés des entreprises sous-traitantes sont, lors des opérations de carénage et de démantèlement des installations, les vrais spécialistes et détenteurs de savoir-faire pour ces phases délicates et complexes.
} 
Auffray A. (21 mars 2011). "Dans les centrales françaises, le malaise de la viande à radiations". Libération.

Bergé C. (2010). Superphenix, deconstruction d'un mythe. Paris, La Découverte, $149 \mathrm{p}$.

Delage C., Guigueno V. (2004). L'historien et le film. Paris, Gallimard, 362 p.

Ferro M. (1993). Cinéma et histoire. Paris, Gallimard, 287 p.

Ferro M., Planchais J. (1997). Les médias et l'histoire, le poids du passé dans le chaos de l'actualité. Paris, CFPJ, $166 \mathrm{p}$.

Filhol E. (2010). La Centrale. Paris, POL Editeur, 132 p.

Hecht G. (2004). Le rayonnement de la France, énergie nucléaire et identité nationale après la Seconde Guerre mondiale. Paris, Editions de La Découverte, 385 p.

Martin C., Portelli A., Guarnieri F. (2013). "Myths and Representations in French Nuclear History: Their Impact on Decommissioning Safety". 22nd ESREL 2013 Conference - Safety, Reliability and Risk Analysis: Beyond the Horizon, Amsterdam.

Martin C., Guarnieri F. (2012). "Nuclear decommissioning and organizational reliability, involving subcontractors in collective action". SFEN.

Mayhew C., Quinlan M. (1997). «The management of health and safety where sub-contractors are employed". Journal of Occupational Health and Safety, Australia.

Michel A., (2009). "Nucléaire, fantasmes et émotions". RGN, $n^{\circ} 2$, mars-avril, p. 29-36.

Nouchi F. (26 août 2013). "Rebecca Zlotowski : Mon film est politique, il n'est pas militant”. Le Monde.

Puiseux H. (1987). L’apocalypse nucléaire et son cinéma. Paris : Les Editions du Cerf, 235 p.

Thébaud-Mony A. (2000). Sous-traitance et servitude. Paris, Inserm, 272 p.

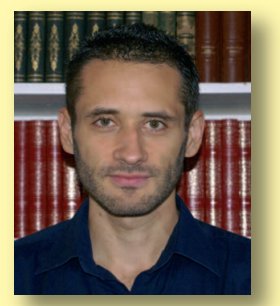

Aurélien PORTELLI est historien des sciences et des techniques. Il est diplômé de l'université de Nice Sophia Antipolis. II est enseignant chercheur à l'ESAIP et chercheur associé au Centre de recherche sur les Risques et les Crises (CRC) de MINES ParisTech. II conduit des recherches sur les mythes et les représentations de l'industrie du nucléaire. II collabore actuellement avec le CEA et son centre de Marcoule dans le cadre d'un projet sur la création et le développement du Service de Protection contre les Rayonnements ionisants (SPR). 